\title{
Parameter Convergence with an Adaptive Output Regulation Problem
}

\author{
Lu Liu, Zhiyong Chen and Jie Huang
}

\begin{abstract}
The global robust output regulation problem for output feedback systems with unknown exosystems was studied some years ago using the adaptive control technique. However, a critical issue concerning the convergence of an estimated parameter vector to the true parameter vector has not been addressed. In this paper, we will address this issue and show that the estimated parameter vector converges to the true parameter vector if the dimension of the internal model is minimal in certain sense.
\end{abstract}

Index Terms-adaptive control, output regulation, nonlinear systems.

\section{INTRODUCTION}

Consider the class of uncertain nonlinear systems described as follows:

$$
\begin{aligned}
\dot{z} & =F(w) z+G(y, v, w) y+D_{1}(v, w) \\
\dot{y} & =H(w) z+K(y, v, w) y+b(w) \xi_{1}+D_{2}(v, w) \\
\dot{\xi}_{i} & =-\lambda_{i} \xi_{i}+\xi_{i+1}, \quad i=1, \cdots, r-2 \\
\dot{\xi}_{r-1} & =-\lambda_{r-1} \xi_{r-1}+u \\
e & =y-q(v, w) \\
\dot{v} & =A_{1}(\sigma) v
\end{aligned}
$$

where $\operatorname{col}(z, y) \in R^{n}$ and $\xi=\operatorname{col}\left(\xi_{1}, \cdots, \xi_{r-1}\right) \in R^{r-1}$ are the states, $y \in R$ is the output, $u \in R$ the input, $e \in R$ the tracking error, $w \in R^{n_{w}}$ the uncertain parameter, and $v \in R^{q}$ the exogenous signal. The signal $v$ represents either the disturbance or reference input or both and is generated by system (2) which is called exosystem. The entries of the matrix $A_{1}(\sigma)$ are functions of a parameter $\sigma \in R^{\sigma}$ where $\Sigma$ is a subset of $R^{\sigma}$. It is assumed that all the functions in system (1) are sufficiently smooth and $D_{1}(0, w)=0, D_{2}(0, w)=0$, and $q(0, w)=0$ for all $w \in R^{n_{w}}$. The system described by (1) and (2) is obtained by performing dynamic extension and coordinate transformation on the class of nonlinear systems in output feedback form ([7], Ch.7). Therefore, for convenience, we will also call the system described by (1) and (2) as an output feedback system.

To have our problem well posed, we assume the following:

Assumption 1.1: For all $\sigma \in \Sigma \subset R^{\sigma}$, all the eigenvalues of $A_{1}(\sigma)$ are distinct with zero real parts.

L. Liu and J. Huang are with the Department of Mechanical and Automation Engineering, The Chinese University of Hong Kong, Shatin, N. T., Hong Kong, China. Z. Chen is with the School of Electrical Engineering and Computer Science, The University of Newcastle, Callaghan, NSW 2308, Australia. (Corresponding author: J. Huang, Tel: (852)26098473, Fax: (852)26036002, E-mail: jhuang@ mae.cuhk.edu.hk).

The work of the second author was partially supported by the Australian Research Council under grant No. DP0878724. The work of the third author was substantially supported by the Research Grants Council of the Hong Kong Special Administration Region under grant No. CUHK412006.
As a result, the general solution of the exosytem is a sum of finitely many sinusoidal functions with their frequencies depending on the eigenvalues of $A_{1}(\sigma)$ and amplitudes and initial phases on the initial condition of $v$.

Briefly, our problem can be stated as follows. Given $\Sigma$, design an output feedback control law such that, for all $w$, all $v(0)$, all $\sigma \in \Sigma$, the trajectory of the closed-loop system starting from any initial state of the plant and the controller exists and is bounded for all $t \geq 0$, and furthermore, the tracking error $e(t)$ approaches zero asymptotically.

The above problem includes the case where the exosystem is known as a special case by letting $\Sigma$ consist of a single known parameter $\sigma$. For this scenario, the global robust output regulation problem for the output feedback systems has been studied in [12] for a special case, and then in [3] for more general case. When the exosystem is unknown, some special version of the global robust output regulation problem for output feedback systems is studied in [4] and [5]. Also, the global robust output regulation problem for a class of large-scale systems was studied in [14]. The largescale system in [14] includes the output feedback system as a special case when the number of the subsystems is equal to one. When the exosystem is unknown, the adaptive control technique has to be adopted to handle a parameter vector which depends on the unknown parameter $\sigma$ and whose dimension is equal to that of the internal model employed. It is important to know whether or not the estimated parameter vector will converge to the true parameter vector. However, this critical issue was not addressed in [14]. In this paper, we will address this parameter convergence problem. We will also introduce the concept of minimal internal model which is an internal model with its dimension being no more than twice as many as the sinusoids in the steady-state input of the system. Then we will show that the estimated parameter vector will converge to the true parameter vector if the minimal internal model is employed.

It should be noted that the output regulation problem for some other classes of nonlinear systems with uncertain exosystem have also been studied in [2], [10], [11], [13]. However, these papers either did not address the parameter convergence issue or did not address this issue thoroughly.

The rest of this paper is organized as follows. Some assumptions and preliminaries will be given in Section II. In Section III, the solvability of the problem will be presented. Section IV further addresses the parameter convergence issue. An example to illustrate our method will be presented in Section V. 


\section{Problem Formulation ANd PReliminaries}

Like in [14], the output regulation of (1) as described above can be converted into an adaptive regulation problem of an augmented system defined in (7). To introduce this conversion, let us list a few additional assumptions.

Assumption 2.1: $b(w)>0$ for all $w \in R^{n_{w}}$.

Assumption 2.2: There exists a sufficiently smooth function $\mathbf{z}(v, w, \sigma)$ with $\mathbf{z}(0,0,0)=0$, such that, for all $v \in R^{q}$, $w \in R^{n_{w}}, \sigma \in \Sigma$,

$$
\begin{aligned}
\frac{\partial \mathbf{z}(v, w, \sigma)}{\partial v} A_{1}(\sigma) v= & F(w) \mathbf{z}(v, w, \sigma) \\
& +G(q(v, w), v, w) q(v, w)+D_{1}(v, w) .
\end{aligned}
$$

Assumption 2.3: There exist an integer $s$, a sufficiently smooth function $\tau: R^{q+n_{w}+\sigma} \mapsto R^{s}$ vanishing at the origin, and a pair of matrices $\Phi(\sigma) \in R^{s \times s}$ and $\Gamma_{1}(\sigma) \in R^{1 \times s}$ where, for all $\sigma \in \Sigma,\left(\Gamma_{1}(\sigma), \Phi(\sigma)\right)$ is observable and all the eigenvalues of $\Phi(\sigma)$ are distinct with zero real parts, such that, for all $v, w$ and $\sigma \in \Sigma$,

$$
\begin{aligned}
& \frac{d \tau(v, w, \sigma)}{d t}=\Phi(\sigma) \tau(v, w, \sigma) \\
& \boldsymbol{\Xi}_{1}(v, w, \sigma)=\Gamma_{1}(\sigma) \tau(v, w, \sigma) .
\end{aligned}
$$

Remark 2.1: As explained in [3], Assumptions 2.1 and 2.2 guarantee that the regulator equations associated with system (1) have a global solution given by $\operatorname{col}(\mathbf{z}(v, w, \sigma), \mathbf{y}(v, w), \boldsymbol{\Xi}(v, w, \sigma))$ and $\mathbf{u}(v, w, \sigma)$, where

$$
\begin{aligned}
\mathbf{y}(v, w)= & q(v, w) \\
\mathbf{\Xi}_{1}(v, w, \sigma)= & \frac{1}{b(w)}\left(\frac{\partial q(v, w)}{\partial v} A_{1}(\sigma) v-H(w) \mathbf{z}(v, w, \sigma)\right. \\
& \left.-K(q(v, w), v, w) q(v, w)-D_{2}(v, w)\right) \\
\mathbf{\Xi}_{i}(v, w, \sigma)= & \frac{\partial \mathbf{\Xi}_{i-1}(v, w, \sigma)}{\partial v} A_{1}(\sigma) v \\
& +\lambda_{i-1} \mathbf{\Xi}_{i-1}(v, w, \sigma), \quad i=2, \cdots, r-1 \\
\mathbf{u}(v, w, \sigma)= & \frac{\partial \mathbf{\Xi}_{r-1}(v, w, \sigma)}{\partial v} A_{1}(\sigma) v \\
& +\lambda_{r-1} \mathbf{\Xi}_{r-1}(v, w, \sigma)
\end{aligned}
$$

and $\boldsymbol{\Xi}(v, w, \sigma)=\operatorname{col}\left(\boldsymbol{\Xi}_{1}(v, w, \sigma), \cdots, \boldsymbol{\Xi}_{r-1}(v, w, \sigma)\right)$.

System (3) is called a steady-state generator with output $\xi_{1}$ of system (1). Since, for all $\sigma \in \Sigma,\left(\Gamma_{1}(\sigma), \Phi(\sigma)\right)$ is observable and all the eigenvalues of $\Phi(\sigma)$ have zero real parts, for any Hurwitz matrix $M \in R^{s \times s}$ and $N \in R^{s \times 1}$ such that $(M, N)$ is controllable, the Sylvester equation $T(\sigma) \Phi(\sigma)-M T(\sigma)=N \Gamma_{1}(\sigma)$ has a unique solution $T(\sigma)$ which is nonsingular. Let $\theta=T(\sigma) \tau$. Then, we can define a dynamic system of the form

$$
\dot{\eta}=M \eta+N \xi_{1} .
$$

System (5) is called an internal model of (1) with output $\xi_{1}$, see, e.g., [3], [8].

Attaching (5) to (1) leads to what is called the augmented system [3]. Further, performing on the augmented system the following coordinate and input transformation

$$
\begin{aligned}
\bar{z} & =z-\mathbf{z}(v, w, \sigma) \\
e & =y-q(v, w) \\
\tilde{\eta} & =\eta-\theta(v, w, \sigma)-N b^{-1}(w) e
\end{aligned}
$$

leads to a lower triangular system of the form

$$
\begin{aligned}
\dot{\bar{z}}= & F(w) \bar{z}+\tilde{G}\left(x_{1}, \mu\right) x_{1} \\
\dot{\tilde{\eta}}= & M \tilde{\eta}+M N b^{-1}(w) x_{1}-N b^{-1}(w)(H(w) \bar{z} \\
& \left.+\tilde{K}\left(x_{1}, \mu\right) x_{1}\right) \\
\dot{x}_{1}= & H(w) \bar{z}+\tilde{K}\left(x_{1}, \mu\right) x_{1}+\Psi^{\sigma} N x_{1}+b(w) \Psi^{\sigma} \tilde{\eta} \\
& +b(w)\left(x_{2}-\Psi^{\sigma} \eta\right) \\
\dot{x}_{i}= & -\lambda_{i-1} x_{i}+x_{i+1}, i=2, \cdots, r
\end{aligned}
$$

where $x=\operatorname{col}\left(x_{1}, \cdots, x_{r}\right)=\operatorname{col}\left(e, \xi_{1}, \cdots, \xi_{r-1}\right), x_{r+1}=$ $u, \mu=\operatorname{col}(v, w), \Psi^{\sigma}=\Gamma_{1}(\sigma) T^{-1}(\sigma)$, and

$$
\begin{aligned}
\tilde{G}\left(x_{1}, \mu\right) x_{1} & =G\left(q+x_{1}, \mu\right)\left(q+x_{1}\right)-G(q, \mu) q \\
\tilde{K}\left(x_{1}, \mu\right) x_{1} & =K\left(q+x_{1}, \mu\right)\left(q+x_{1}\right)-K(q, \mu) q .
\end{aligned}
$$

Remark 2.2: System (7) is called a semi-translated augmented system which is somehow different from the translated augmented system (15)-(19) described in [3] in that no coordinates transform is performed on the states $x_{2}, \cdots, x_{r}$ and no input transform on $u$. Also, due to the explicit presence of $\eta$ which can be viewed as a persistent external input to system (7), the origin is not an equilibrium of system (7). As a result, we cannot solve the output regulation problem of our original system by stabilizing (7). Nevertheless, it can still be seen that, if there exists a control law of the form

$$
\begin{aligned}
\dot{\zeta} & =g_{\zeta}\left(\zeta, x_{1}, \cdots, x_{r}, \eta\right) \\
u & =k_{\zeta}\left(\zeta, x_{1}, \cdots, x_{r}, \eta\right)
\end{aligned}
$$

that solves the global robust regulation problem of system (7) in the sense that, for any initial condition of the closed-loop system and the exosystem, and any fixed unknown parameter $w$ and $\sigma$, the solution of the closed-loop system is bounded for all $t \geq 0$, and $x_{1}$ approaches 0 asymptotically, then the following control law

$$
\begin{aligned}
\dot{\eta} & =M \eta+N \xi_{1} \\
\dot{\zeta} & =g_{\zeta}\left(\zeta, e, \xi_{1}, \cdots, \xi_{r-1}, \eta\right) \\
u & =k_{\zeta}\left(\zeta, e, \xi_{1}, \cdots, \xi_{r-1}, \eta\right)
\end{aligned}
$$

solves the global robust output regulation of system (1).

In what follows, we will focus on solving the global robust regulation problem for system (7). To this end, we further assume the following:

Assumption 2.4: $F(w)$ is Hurwitz for all $w \in R^{n_{w}}$.

\section{Solvability of the Problem}

Let us first introduce a few inequalities and notations to be used later. Since $\tilde{G}\left(x_{1}, \mu\right)$ and $\tilde{K}\left(x_{1}, \mu\right)$ are realvalued continuous functions, there exist sufficiently smooth 
functions $q_{i}(\mu) \geq 1, a_{i}\left(x_{1}\right) \geq 1, i=1,2$, such that, for each $\mu \in R^{q} \times R^{n_{w}}, x_{1} \in R$,

$$
\begin{aligned}
\left|\tilde{G}\left(x_{1}, \mu\right) x_{1}\right|^{2} & \leq q_{1}(\mu) a_{1}\left(x_{1}\right) x_{1}^{2} \\
\left|\tilde{K}\left(x_{1}, \mu\right) x_{1}\right|^{2} & \leq q_{2}(\mu) a_{2}\left(x_{1}\right) x_{1}^{2} .
\end{aligned}
$$

Define $\bar{x}_{1}=x_{1}, \bar{x}_{r+1}=0, \gamma_{0}=0$,

$$
\begin{aligned}
\alpha_{1}\left(x_{1}, k, \eta, \hat{\Psi}\right) & =-k \rho\left(\bar{x}_{1}\right) \bar{x}_{1}+\hat{\Psi} \eta \\
\tau_{1}\left(x_{1}, \eta\right) & =-\eta^{T} \bar{x}_{1} \\
\bar{x}_{i+1} & =x_{i+1}-\alpha_{i}, \quad i=1, \cdots, r \\
\gamma_{i-1}\left(x_{1}, \cdots, x_{i}, k, \eta, \hat{\Psi}, \hat{b}\right) & =\gamma_{i-2}-\frac{\partial \alpha_{i-1}}{\partial \bar{x}_{1}} \bar{x}_{i}\left(x_{2}-\hat{\Psi} \eta\right)
\end{aligned}
$$

with $\rho(\cdot)$ being some smooth nonnegative function to be specified later. Further, for $i=2, \cdots, r$, let

$$
\begin{aligned}
& \alpha_{i}\left(x_{1}, \cdots, x_{i}, k, \eta, \hat{\Psi}, \hat{b}\right) \\
& =\quad \lambda_{i-1} x_{i}+\frac{\partial \alpha_{i-1}}{\partial k} \dot{k}+\sum_{j=2}^{i-1} \frac{\partial \alpha_{i-1}}{\partial x_{j}} \dot{x}_{j} \\
& +\frac{\partial \alpha_{i-1}}{\partial \eta} \dot{\eta}+\frac{\partial \alpha_{i-1}}{\partial \hat{b}} \gamma_{i-1}\left(x_{1}, \cdots, x_{i}, k, \eta, \hat{\Psi}, \hat{b}\right) \\
& +\left(\hat{b}-\sum_{j=2}^{i-2} \bar{x}_{j+1} \frac{\partial \alpha_{j}}{\partial \hat{b}}\right) \frac{\partial \alpha_{i-1}}{\partial \bar{x}_{1}}\left(x_{2}-\hat{\Psi} \eta\right) \\
& -\bar{x}_{i-1}-\bar{x}_{i}-\left(\frac{\partial \alpha_{i-1}}{\partial \bar{x}_{1}}\right)^{2} \bar{x}_{i}+\tau_{i} \frac{\partial \alpha_{i-1}}{\partial \hat{\Psi}} \\
& +\frac{\partial \alpha_{i-1}}{\partial \bar{x}_{1}} \eta^{T} \sum_{j=1}^{i-2} \frac{\partial \alpha_{j}}{\partial \hat{\Psi}} \bar{x}_{j+1} \\
& \tau_{i}\left(x_{1}, \cdots, x_{i}, k, \eta, \hat{\Psi}, \hat{b}\right)=\tau_{i-1}+\frac{\partial \alpha_{i-1}}{\partial \bar{x}_{1}} \eta^{T} \bar{x}_{i}(.10)
\end{aligned}
$$

Lemma 3.1: Consider the closed-loop system composed of (7) and the following control law

$$
\begin{aligned}
u & =\alpha_{r}(x, k, \eta, \hat{\Psi}, \hat{b}) \\
\dot{k} & =\rho\left(\bar{x}_{1}\right) \bar{x}_{1}^{2} \\
\dot{\hat{\Psi}} & =\tau_{r}(x, k, \eta, \hat{\Psi}, \hat{b}) \\
\dot{\hat{b}} & =\gamma_{r-1}(x, k, \eta, \hat{\Psi}, \hat{b}) .
\end{aligned}
$$

There exist a sufficiently smooth function $\rho$ and a Lyapunov function $V_{r}$ such that, along the trajectory of the closed-loop system,

$$
\dot{V}_{r} \leq-\|\bar{z}\|^{2}-\|\tilde{\eta}\|^{2}-\sum_{j=1}^{r} \bar{x}_{j}^{2} .
$$

\section{Proof: Let}

$$
\begin{aligned}
V_{1}= & 2 \bar{l}^{T} \tilde{z}(w) \bar{z}+2 \bar{h} \tilde{\eta}^{T} P \tilde{\eta}+\bar{x}_{1}^{2}+b(w)(k-\bar{k})^{2} \\
& +b(w)\left\|\hat{\Psi}-\Psi^{\sigma}\right\|^{2}
\end{aligned}
$$

with $\bar{l}, \bar{h}$, and $\bar{k}$ some positive constants and $\tilde{P}(w)$ and $P$ the positive definite solutions to the Lyapunov equations

$$
\begin{aligned}
& \tilde{P}(w) F(w)+F^{T}(w) \tilde{P}(w)=-I_{n-1} \\
& P M+M^{T} P=-I_{s} .
\end{aligned}
$$

Then, the time derivative of $V_{1}$ along the trajectory of $\bar{x}_{1}$ subsystem with $x_{2}=\bar{x}_{2}+\alpha_{1}$ is given by

$$
\begin{array}{r}
\dot{V}_{1} \leq-2\left(l-\|H(w)\|^{2}\right)\|\bar{z}\|^{2}-2\left(h-\left\|\Psi^{\sigma} b(w)\right\|^{2}\right)\|\tilde{\eta}\|^{2} \\
+2\left(\bar{q}_{1}(\mu) s_{1}\left(\bar{x}_{1}\right)+q_{2}(\mu) a_{2}\left(\bar{x}_{1}\right)+\left\|\Psi^{\sigma} N\right\|^{2}\right. \\
\left.-b(w) \bar{k} \rho\left(\bar{x}_{1}\right)\right) \bar{x}_{1}^{2}+2 b(w)\left(\hat{\Psi}-\Psi^{\sigma}\right)\left(\dot{\hat{\Psi}}-\tau_{1}\right)^{T} \\
+2 b(w) \bar{x}_{1} \bar{x}_{2}
\end{array}
$$

where $l=\bar{l}\left(1-\epsilon_{1}\|\tilde{P}(w)\|^{2}\right)-\bar{h} \epsilon_{2}^{-1}\|H(w)\|^{2}, \quad h=$ $\bar{h}\left(1-\epsilon_{2}\left\|P M N b^{-1}(w)\right\|^{2}-2 \epsilon_{2}\left\|P N b^{-1}(w)\right\|^{2}\right), \quad \bar{q}_{1}(\mu) \geq$ $\max \left(\bar{l} \epsilon_{1}^{-1} q_{1}(\mu), \bar{h} \epsilon_{2}^{-1} q_{2}(\mu), 1\right)$ and $s_{1}\left(\bar{x}_{1}\right)=a_{1}\left(\bar{x}_{1}\right)+$ $a_{2}\left(\bar{x}_{1}\right)+2, \forall \epsilon_{1}>0, \forall \epsilon_{2}>0$.

Letting

$$
V_{i}=V_{1}+\sum_{j=2}^{i} \bar{x}_{j}^{2}+(\hat{b}-b(w))^{2}
$$

gives

$$
\begin{array}{r}
\dot{V}_{i} \leq-2\left(l-i\|H(w)\|^{2}\right)\|\bar{z}\|^{2}-2\left(h-i\left\|\Psi^{\sigma} b(w)\right\|^{2}\right)\|\tilde{\eta}\|^{2} \\
+2\left(\bar{q}(\mu) s\left(\bar{x}_{1}\right)+i\left(q_{2}(\mu) a_{2}\left(\bar{x}_{1}\right)+\left\|\Psi^{\sigma} N\right\|^{2}\right)\right. \\
\left.-b(w) \bar{k} \rho\left(\bar{x}_{1}\right)\right) \bar{x}_{1}^{2}+2\left(\hat{b}-b(w)-\sum_{j=2}^{i-1} \bar{x}_{j+1} \frac{\partial \alpha_{j}}{\partial \hat{b}}\right) \\
\times\left(\dot{\hat{b}}-\gamma_{i-1}\left(x_{1}, \cdots, x_{i}, k, \eta, \hat{b}\right)\right) \\
+2\left(b(w) \hat{\Psi}-b(w) \Psi^{\sigma}-\sum_{j=1}^{i-1} \bar{x}_{j+1}\left(\frac{\partial \alpha_{j}}{\partial \hat{\Psi}}\right)^{T}\right) \\
\times\left(\dot{\hat{\Psi}}-\tau_{i}\right)^{T}-\sum_{j=2}^{i} \bar{x}_{j}^{2}+2 \bar{x}_{i} \bar{x}_{i+1}
\end{array}
$$

where $\bar{q}(\mu) \geq \max \left(\bar{q}_{1}(\mu), b(w)^{2}+1\right), s\left(\bar{x}_{1}\right) \geq s_{1}\left(\bar{x}_{1}\right)+1$.

Finally, by taking the control law (11) we obtain

$$
\begin{array}{r}
\dot{V}_{r} \leq-2\left(l-r\|H(w)\|^{2}\right)\|\bar{z}\|^{2}-2\left(h-r\left\|\Psi^{\sigma} b(w)\right\|^{2}\right)\|\tilde{\eta}\|^{2} \\
+2\left(\bar{q}(\mu) s\left(\bar{x}_{1}\right)+r\left(q_{2}(\mu) a_{2}\left(\bar{x}_{1}\right)+\left\|\Psi^{\sigma} N\right\|^{2}\right)\right. \\
\left.-b(w) \bar{k} \rho\left(\bar{x}_{1}\right)\right) \bar{x}_{1}^{2}-\sum_{j=2}^{r} \bar{x}_{j}^{2} .
\end{array}
$$

For any fixed $w$ and $\sigma$, there exist $\epsilon_{1}, \epsilon_{2}$ such that $1-\epsilon_{2}\left\|P M N b_{\tilde{P}}^{-1}(w)\right\|^{2}-2 \epsilon_{2}\left\|P N b^{-1}(w)\right\|^{2}>0$, and $1-\epsilon_{1}\|\tilde{P}(w)\|^{2}>0$. Thus, if we choose $\bar{h} \geq \frac{1+r\left\|\Psi^{\sigma} b(w)\right\|^{2}}{1-\epsilon_{2}\left\|P M N b^{-1}(w)\right\|^{2}-2 \epsilon_{2}\left\|P N b^{-1}(w)\right\|^{2}}, \quad \bar{l} \geq$ $\frac{1+r\|H(w)\|^{2}+\bar{h} \epsilon_{2}^{-1}\|H(w)\|^{2}}{1-\epsilon_{1}\|\tilde{P}(w)\|^{2}}$, then $l-r\|H(w)\|^{2} \geq$ $1, h-r\left\|\Psi^{\sigma} b(w)\right\|^{2} \geq 1$. Also, letting $\bar{k} \geq$ $\frac{1}{a b(w)} \max \left(\bar{q}(\mu), r q_{2}(\mu), r\left\|\Psi^{\sigma} N\right\|^{2}\right), \rho\left(\bar{x}_{1}\right) \geq a\left(s\left(\bar{x}_{1}\right)+\right.$ $\left.a_{2}\left(\bar{x}_{1}\right)+2\right)$ with $a$ any positive real number yields $b(w) \bar{k} \rho\left(\bar{x}_{1}\right) \geq \bar{q}(\mu) s\left(\bar{x}_{1}\right)+r\left(q_{2}(\mu) a_{2}\left(\bar{x}_{1}\right)+\left\|\Psi^{\sigma} N\right\|^{2}\right)+1$.

As a result, we obtain inequality (12).

We now make use of the Lyapunov function candidate $V_{r}$ and inequality (12) to establish the following result.

Theorem 3.1: Under Assumptions 1.1, 2.1-2.4, there exists a feedback controller composed of (5) and (11) that 
solves the global robust output regulation problem for nonlinear system (1) with exosystem (2).

Proof: Let $\bar{x}_{c}=\left(\bar{z}^{T}, \tilde{\eta}^{T}, \bar{x}_{1}, \cdots, \bar{x}_{r}, k, \hat{\Psi}, \hat{b}\right)^{T}$. Since $V_{r}$ is positive definite and thus inequality (12) implies that $\bar{x}_{c}$ is bounded for all $t \geq 0$. Using the definition of $\tilde{\eta}$ shows $\eta$ is also bounded since $v$ is bounded. Thus, $\alpha_{i}$ as well as $x_{i}$, $i=1, \cdots, r$, are bounded. Therefore, all the states as well as their derivatives of the closed-loop system composed of (7) and (11) are bounded. Furthermore, using (12) shows $\bar{z}$, $\tilde{\eta}$ and $\bar{x}_{i}, 1 \leq i \leq r$, are square integrable on $[0, \infty)$. By Barbalat's lemma, $\operatorname{col}\left(\bar{z}, \tilde{\eta}, \bar{x}_{1}, \cdots, \bar{x}_{r}\right)$ approaches zero as $t \rightarrow \infty$. Thus the proof is completed.

Remark 3.1: It can be seen that the closed-loop system composed of the given system (1), exosystem (2) and the control law (5) and (11) has the following two properties:

(i) $\lim _{t \rightarrow \infty}\left(x_{2}-\Psi^{\sigma} \eta\right)=0$,

(ii) $\lim _{t \rightarrow \infty} \dot{\hat{\Psi}}=0$ and

$$
\lim _{t \rightarrow \infty}\left(\hat{\Psi}-\Psi^{\sigma}\right) T(\sigma) \tau=0 .
$$

In fact, from the third equation of (7), the first property follows from the fact that $\dot{x}_{1}, x_{1}, \bar{z}$, and $\tilde{\eta}$ approach zero as $t \rightarrow \infty$. Also, from (11), $\dot{\hat{\Psi}}$ is a linear function of $\bar{x}_{1}, \cdots, \bar{x}_{r}$. Thus $\lim _{t \rightarrow \infty} \dot{\hat{\Psi}}=0$ since $\lim _{t \rightarrow \infty} \bar{x}_{i}=0, i=1, \cdots, r$. To show (15), note that since both $\bar{x}_{1}$ and $\bar{x}_{2}$ approach 0 as $t \rightarrow \infty$, so does $\left(x_{2}-\hat{\Psi} \eta\right)$. Further, noting $\left(x_{2}-\Psi^{\sigma} \eta\right)$ approaches zero as $t \rightarrow \infty$ gives

$$
\lim _{t \rightarrow \infty}\left(\hat{\Psi}-\Psi^{\sigma}\right) \eta=0
$$

Finally, noting $\lim _{t \rightarrow \infty}(\eta-\theta)=0$ and $\theta=T(\sigma) \tau$ gives (15).

\section{CONVERGENCE OF $\hat{\Psi}$ TO $\Psi^{\sigma}$ AND THE MiNimaL INTERNAL MODEL}

In this section, we will ascertain conditions under which the unknown parameter vector $\hat{\Psi}$ will converge to $\Psi^{\sigma}$ as $t$ tends to infinity. For this purpose, let us first establish a lemma.

Lemma 4.1: Let $g:[0, \infty) \rightarrow R^{n}$ be continuously differentiable and $f:[0, \infty) \rightarrow R^{n}$ be bounded piecewise continuous function. Further assume there exist positive constants $\epsilon, t_{0}, T_{0}$ such that, for any unit row vector $\mathbf{c}$ of dimension $n$, and any $t \geq t_{0}$,

$$
\frac{1}{T_{0}} \int_{t}^{t+T_{0}}|\mathbf{c} f(s)| d s \geq \epsilon .
$$

Then

$$
\lim _{t \rightarrow \infty} g(t)=0
$$

if

$$
\lim _{t \rightarrow \infty} \dot{g}(t)=0
$$

and

$$
\lim _{t \rightarrow \infty} g^{T}(t) f(t)=0
$$

Proof: Suppose (17) is not true. Then there exist a time sequence $s_{1}<s_{2}<\cdots$ satisfying $s_{i} \rightarrow \infty$ as $i \rightarrow \infty$ and a number $\delta_{1}>0$, such that $\left|g\left(s_{i}\right)\right|>\delta_{1}$.

By assumptions (18) and (19), for any $\delta_{2}>0$ and $\delta_{3}>0$, there exists a time $t_{1}$, such that,

$$
|\dot{g}(t)| \leq \delta_{2}, \quad \forall t \geq t_{1}
$$

and

$$
\left|g^{T}(t) f(t)\right| \leq \delta_{3}, \quad \forall t \geq t_{1}
$$

As a result,

$$
\left|g\left(t+T_{0}\right)-g(t)\right| \leq \int_{t}^{t+T_{0}}|\dot{g}(x)| d x \leq \delta_{2} T_{0}, \forall t>t_{1} .
$$

Let $\bar{f}$ be some real number such that $|f(t)|<\bar{f}, \forall t \geq 0$. Then, for any $s_{i}>t_{1}$,

$$
\begin{aligned}
& \int_{s_{i}}^{s_{i}+T_{0}}\left|g^{T}(s) f(s)\right| d s \\
\geq & \int_{s_{i}}^{s_{i}+T_{0}}\left|g^{T}\left(s_{i}\right) f(s)\right| d s \\
& -\int_{s_{i}}^{s_{i}+T_{0}}\left|\left[g(s)-g\left(s_{i}\right)\right]^{T} f(s)\right| d x \\
\geq & T_{0} \delta_{1} \epsilon-\delta_{2} T_{0} \bar{f} .
\end{aligned}
$$

Since $\delta_{2}$ can be arbitrarily small, $\int_{s_{i}}^{s_{i}+T_{0}}\left|g^{T}(s) f(s)\right| d s \geq \epsilon_{2}$ for some positive $\epsilon_{2}$ independent of $\delta_{3}$. Thus, there exists a time $\bar{s}_{i} \in\left[s_{i}, s_{i}+T_{0}\right]$ such that

$$
\left|g^{T}\left(\bar{s}_{i}\right) f\left(\bar{s}_{i}\right)\right| \geq \epsilon_{2} / T_{0} .
$$

Noting $\delta_{3}$ can be arbitrarily small leads to a contradiction. The proof is thus completed.

Remark 4.1: Condition (16) is known as persistent excitation (PE) condition [9]. This lemma gives the convergence condition of the function $g(t)$ to the origin based on two asymptotic properties (18) and (19) of $g(t)$ and the persistent excitation condition of $f(t)$. The result is of interest in that it does not assume that $g(t)$ has to be governed by some linear differential equation as assumed in the literature of adaptive control of linear systems. Thus, it also applies to adaptive control of nonlinear systems. It should be noted that the fact that $\lim _{t \rightarrow \infty} \dot{g}(t)=0$ alone plus the PE condition of $f$ does not necessarily imply the existence of $\lim _{t \rightarrow \infty} g(t)$.

We are now ready to introduce the concept of minimal internal model to give the conditions under which $\lim _{t \rightarrow \infty}\left(\hat{\Psi}-\Psi^{\sigma}\right)=0$. For this purpose, we assume, without loss of generality, that $\Phi(\sigma)$ and $\Gamma_{1}(\sigma)$ is in the following observer canonical form:

$$
\begin{aligned}
\Phi & =\left[\begin{array}{ccccc}
0 & 1 & 0 & \cdots & 0 \\
0 & 0 & 1 & \cdots & 0 \\
\vdots & \vdots & \vdots & \cdots & \vdots \\
0 & 0 & 0 & \cdots & 1 \\
a_{1}(\sigma) & a_{2}(\sigma) & a_{3}(\sigma) & \cdots & a_{s}(\sigma)
\end{array}\right] \\
\Gamma_{1} & =\left[\begin{array}{llll}
1 & 0 & \cdots & 0
\end{array}\right]
\end{aligned}
$$


where, for each $\sigma \in R^{\sigma}, a_{1}(\sigma), a_{2}(\sigma), a_{3}(\sigma), \cdots, a_{s}(\sigma)$ are constant real numbers. Thus, $\boldsymbol{\Xi}_{1}(v, w, \sigma)$ satisfies, for all $\sigma \in \Sigma$,

$$
\begin{aligned}
& \frac{d^{s} \boldsymbol{\Xi}_{1}(v, w, \sigma)}{d t^{s}} \\
= & a_{1}(\sigma) \boldsymbol{\Xi}_{1}(v, w, \sigma)+a_{2}(\sigma) \frac{d \boldsymbol{\Xi}_{1}(v, w, \sigma)}{d t}+ \\
\cdots \quad & +a_{s}(\sigma) \frac{d^{(s-1)} \boldsymbol{\Xi}_{1}(v, w, \sigma)}{d t^{(s-1)}}
\end{aligned}
$$

for all trajectories $v(t)$ of the exosystem, and all $w$, and

$$
\begin{aligned}
\tau(v, w, \sigma)= & \operatorname{col}\left(\boldsymbol{\Xi}_{\mathbf{1}}(v, w, \sigma)\right), \dot{\boldsymbol{\Xi}}_{1}(v, w, \sigma), \\
& \left.\cdots, \boldsymbol{\Xi}_{1}^{(s-1)}(v, w, \sigma)\right) .
\end{aligned}
$$

As an elaboration of what was introduced in [6], we call a monic polynomial $P^{\sigma}(\lambda)=\lambda^{s}-a_{1}(\sigma)-a_{2}(\sigma) \lambda-\cdots-$ $a_{s}(\sigma) \lambda^{s-1}$ a global zeroing polynomial of $\boldsymbol{\Xi}_{1}(v, w, \sigma)$ on $\Sigma$, if, along all trajectories $v(t)$ of the exosystem $\dot{v}=A_{1}(\sigma) v$, all $\sigma \in \Sigma$, and all $w, \boldsymbol{\Xi}_{1}(v, w, \sigma)$ satisfies a differential equation of the form (21). A monic polynomial $P^{\sigma}(\lambda)$ is called a minimal zeroing polynomial of $\boldsymbol{\Xi}_{1}(v, w, \sigma)$ on $\Sigma$ if $P(\lambda)$ is a zeroing polynomial of $\boldsymbol{\Xi}_{1}(v, w, \sigma)$ on $\Sigma$ of least degree. Assume $\boldsymbol{\Xi}_{1}(v, w, \sigma)$ is an analytic function in $v$ and the nonzero eigenvalues of $A_{1}$ be $\pm j \omega_{1}, \cdots, \pm j \omega_{k}$ with $\omega_{i}>0$ where $k=q / 2$ if $q$ is even and $k=(q-1) / 2$ if $q$ is odd. Then, it can be deduced from the result of [6] that, there exist a set

$$
\Omega=\left\{l_{1} \omega_{1}+\cdots+l_{k} \omega_{k}, l_{1}, \cdots, l_{k}=0, \pm 1, \cdots, \pm \infty\right\},
$$

an integer $s$, and $s$ distinct members of $\Omega$ denoted by $\hat{\omega}_{l}$, $l=1, \cdots, s$, such that, along all trajectories $v(t)$ of the exosystem, for any $w$ and any $\sigma \in \Sigma$,

$$
\boldsymbol{\Xi}_{1}(v(t), w, \sigma)=\sum_{l=1}^{s} C_{l}\left(v_{0}, w, \sigma\right) e^{j \hat{\omega}_{l} t}
$$

where $C_{l}\left(v_{0}, w, \sigma\right)$ are complex numbers not identically zero for all $v_{0}, w$, and $\sigma$.

We call a steady-state generator as the minimal steadystate generator if the dimension of the steady-state generator (3) is equal to the degree $s$ of the minimal zeroing polynomial of $\boldsymbol{\Xi}_{1}(v, w, \sigma)$ on $\Sigma$. Since the dimension of an internal model is equal to that of the corresponding steadystate generator, we call an internal model as the minimal internal model if the dimension of the internal model is equal to the degree of the minimal polynomial of $\boldsymbol{\Xi}_{1}(v, w, \sigma)$ on $\Sigma$.

We are now ready to state our main conclusion as follows:

Theorem 4.1: Under Assumptions 1.1, 2.1-2.4, suppose $v(t)$ is a periodic function of period $T_{0}$ (which is true if $\omega_{1}, \cdots, \omega_{k}$ have a common multiple). If the internal model (5) is of minimal dimension, and $v_{0}, w$ and $\sigma$ are such that none of $C_{l}\left(v_{0}, w, \sigma\right)$ is zero, then the feedback controller composed of (5) and (11) is such that $\lim _{t \rightarrow \infty}\left(\hat{\Psi}-\Psi^{\sigma}\right)=0$.

Proof: Let $g^{T}(t)=\left(\hat{\Psi}(t)-\Psi^{\sigma}\right) T(\sigma)$ and $f(t)=$ $\tau(v(t), w, \sigma)$. Then both $g$ and $f$ are well defined over
$[0, \infty), g$ is continuously differentiable and $f$ is bounded piecewise continuous over $[0, \infty)$ and periodic with period $T_{0}$. By (15), $g$ and $f$ satisfy conditions (18) and (19), respectively. We now only need to show that $f$ also satisfies (16). Let $\mathbf{c}=\left[c_{1}, \cdots, c_{s}\right]$ be any constant row vector of dimension $s$. Then $\mathbf{c} f(t)$ is a trigonometric polynomial, i.e., it is a linear combination of the sinusoidal functions $\sin \left(\hat{\omega}_{l}+\phi_{l}\right), l=1, \cdots, s$. Moreover, the fact that $\mathbf{c} f(t)=0$ for all $t \geq 0$ implies

$$
c_{1}+c_{2}\left(j \hat{\omega}_{l}\right)+\cdots+c_{s}\left(j \hat{\omega}_{l}\right)^{s-1}=0
$$

for $l=1, \cdots, s$. Thus, $\mathbf{c}$ must be zero. Therefore, for any unit row vector $\mathbf{c}, \mathbf{c} f(t)$ is a nonzero trigonometric polynomial. Consider the following function $\int_{t}^{t+T_{0}}|\mathbf{c} f(s)| d s$ which is a continuous function of $\mathbf{c}$ with a compact domain $\|\mathbf{c}\|=1$, is independent of $T_{0}$ and any $t>0$, and is strictly positive for any unit row vector c. Thus $f$ satisfies (16). The proof is thus completed by Lemma 4.1 .

Remark 4.2: In Theorem 4.1, for the simplicity of proof, we assume the periodic property of $v(t)$. It is possible to remove this assumption by using the argument given in Lemma 3.4 of [1]. The employment of a minimal internal model guarantees that the dimension of the unknown vector $\Psi^{\sigma}$ is no more than twice as many as the sinusoids in the signal $\boldsymbol{\Xi}_{1}$. Thus, the employment of an minimal internal model guarantees the satisfaction of the persistent excitation condition. This condition is indispensable. In fact, if the dimension of the steady-state generator were $\hat{s}>s$ for some integer $\hat{s}$, then $\tau$ would be as follows

$$
\begin{array}{r}
\tau(v, w, \sigma)=\operatorname{col}\left(\boldsymbol{\Xi}_{1}(v, w, \sigma)\right), \dot{\boldsymbol{\Xi}}_{1}(v, w, \sigma), \\
\left.\ldots, \boldsymbol{\Xi}_{1}^{(\hat{s}-1)}(v, w, \sigma)\right) .
\end{array}
$$

Let $\mathbf{c}=\left[c_{1}, \cdots, c_{\hat{s}}\right]$ be any constant row vector of dimension $\hat{s}$. Then the fact that $\mathbf{c} f(t)=0$ for all $t$ would still imply

$$
c_{1}+c_{2}\left(j \hat{\omega}_{l}\right)+\cdots+c_{\hat{s}}\left(j \hat{\omega}_{l}\right)^{\hat{s}-1}=0
$$

for $l=1, \cdots, s$. But (24) would not imply c $=0$ since $\hat{s}>s$. On the other hand, assume the minimal internal model is employed and $\left(v_{0}, w, \sigma\right)$ is such that $C_{l}\left(v_{0}, w, \sigma\right)=0$ for some $l$. Then there exists nonzero $\mathbf{c}=\left[c_{1}, \cdots, c_{s}\right]$ such that $\mathbf{c} f(t)=0$ for all $t$. Thus the assumption that $v_{0}, w$ and $\sigma$ are such that none of $C_{l}\left(v_{0}, w, \sigma\right)$ is zero is also indispensable.

I

\section{AN EXAMPLE}

Consider the system

$$
\begin{aligned}
\dot{z}_{1} & =-z_{1}+\sin ^{2}\left(y-v_{1}\right) y \\
\dot{z}_{2} & =-z_{2}+y \\
\dot{y} & =z_{2}-a_{1} y-a_{3} y^{3}+b \xi_{1} \\
\dot{\xi}_{1} & =-\xi_{1}+u \\
e & =y-v_{1} \\
\dot{v} & =A_{1}(\sigma) v, \quad t \geq 0
\end{aligned}
$$

where $a_{1}$ and $a_{3}$ are nonzero real numbers, $b$ is an unknown positive number, and $A_{1}=\left[\begin{array}{cc}0 & \sigma \\ -\sigma & 0\end{array}\right]$ with $\sigma \in \Sigma=\{a>$ 
$0\}$. System (25) is in the form of (1) with exosystem (2) with $z=\operatorname{col}\left(z_{1}, z_{2}\right), v=\operatorname{col}\left(v_{1}, v_{2}\right), F(w)=\left[\begin{array}{cc}-1 & 0 \\ 0 & -1\end{array}\right]$, $G(y, v, w) y=\left[\begin{array}{c}-\sin ^{2}\left(y-v_{1}\right) y \\ y\end{array}\right], H(w)=\left[\begin{array}{ll}0 & 1\end{array}\right]$, $K(y, v, w) y=-a_{1} y-a_{3} y^{3}, D_{1}(v, w)=D_{2}(v, w)=0, r=$ 2 and $\lambda_{1}=1$. It can be verified that all assumptions needed for the satisfaction of Theorem 3.1 and Theorem 4.1 are satisfied. In particular, the solution of the regulator equations is $\mathbf{z}_{\mathbf{1}}(v, w, \sigma)=0, \mathbf{z}_{\mathbf{2}}(v, w, \sigma)=z_{10} v_{1}+z_{01} v_{2}, \mathbf{y}(v, w)=$ $v_{1}, \boldsymbol{\Xi}_{\mathbf{1}}(v, w, \sigma)=a_{10} v_{1}+a_{01} v_{2}+a_{30} v_{1}^{3}$, where $z_{10}=\frac{1}{1+\sigma^{2}}$, $z_{01}=-\frac{\sigma}{1+\sigma^{2}}, a_{10}=a_{1}-z_{10}, a_{01}=\sigma-z_{01}, a_{30}=a_{3}$. Further, it can be seen that the minimal zero polynomial of $\boldsymbol{\Xi}_{1}(v, w, \sigma)$ is $\lambda^{4}+10 \sigma^{2} \lambda^{2}+9 \sigma^{4}$. Thus the minimal internal model is of dimension 4 ,

Letting $v(0)=\left[\begin{array}{l}v_{10} \\ v_{20}\end{array}\right]$ gives $v(t)=A\left[\begin{array}{l}\sin \left(\sigma t+\phi_{1}\right) \\ \sin \left(\sigma t+\phi_{2}\right)\end{array}\right]$ with $A=\sqrt{v_{10}^{2}+v_{20}^{2}}, \phi_{1}=\arctan \frac{v_{10}}{v_{20}}$, and $\phi_{2}=$ $\arctan \left(-\frac{v_{20}}{v_{10}}\right)$. As a result,

$$
\begin{aligned}
\mathbf{\Xi}_{1}(v(t), w, \sigma) & =a_{10} v_{1}+a_{01} v_{2}+a_{30} v_{1}^{3} \\
& =P_{1}^{3} \sin \left(\sigma t+\psi_{1}^{3}\right)+P_{3}^{3} \sin \left(3 \sigma t+\psi_{3}^{3}\right)
\end{aligned}
$$

with $P_{1}^{3}=\left(\left(a_{10} A+\frac{3}{4} a_{30} A^{3}\right)^{2}+a_{01}^{2} A^{2}+a_{01} A^{2}\left(2 a_{10}+\right.\right.$ $\left.\left.\frac{3}{2} a_{30} A^{2}\right) \cos \left(\phi_{2}-\phi_{1}\right)\right)^{\frac{1}{2}}$ and $P_{3}^{3}=-\frac{1}{4} a_{30} A^{3}$, which implies that $\boldsymbol{\Xi}_{1}(v(t), w, \sigma)$ is the sum of two sinusoidal functions with frequencies $\sigma$ and $3 \sigma$. Since the internal model is minimal, and for all nonzero $v(0), w$, and any $\sigma \in \Sigma$, the amplitudes of the two sinusoidal functions are nonzero. The condition for the convergence of the unknown parameter $\hat{\Psi}$ to the true value $\Psi^{\sigma}$ is also satisfied.

Following the procedure of Section III, a specific controller can be designed. Due to the space limit, the details are omitted. Computer simulation is also conducted and some results are shown in Figures 1-2 where the initial condition is $v_{1}(0)=1, v_{2}(0)=0, z_{1}(0)=1, z_{2}(0)=0$, $y(0)=1, \xi_{1}(0)=0, \eta(0)=0, \hat{\Psi}(0)=0, k(0)=3$, $\hat{b}(0)=0$, the parameters are $\sigma=0.2, b=1, a=1$, $a_{1}=1, a_{3}=1$. It can be seen that the objective of output regulation is achieved and $\hat{\Psi}$ converges to $\left.\Psi^{\sigma}\right|_{\sigma=0.2}=$ $\left[\begin{array}{llll}1.766 & 1.569 & 0.062 & -0.392\end{array}\right]$.

\section{CONCLUSION}

In this paper, the parameter convergence problem in the output regulation of output feedback systems with unknown exosystem has been addressed. It is emphasized that the result on the convergence of the estimated parameter is not limited to the output feedback systems and it also applies to other types of nonlinear systems.

\section{REFERENCES}

[1] S. Boyd and S. Sastry, "On parameter convergence in adaptive control," Systems and Control Letters, vol. 3, pp. 311-319, 1983.

[2] Z. Chen, and J. Huang, "Global tracking of uncertain nonlinear cascaded systems with adaptive internal model, "Proceedings of 2002 IEEE Conference on Decision and Control, pp. 3855-3862, Las Vegas, Nevada, Dec. 2002.

[3] Z. Chen and J. Huang, "Global robust output regulation for output feedback systems," IEEE Transactions on Automatic Control, vol. 50, pp. 117-121, 2005.
[4] Z. Ding, "Global stabilization and disturbance suppression of a class of nonlinear systems with uncertain internal model," Automatica, vol. 39, pp. 471-479, 2003.

[5] Z. Ding, "Adaptive estimation and rejection of unknown sinusoidal disturbances in a class of non-minimum-phase nonlinear systems," IEE Proc.-Control Theory Appl., vol. 153, no. 4, pp. 379-386, July 2006.

[6] J. Huang, "Remarks on the robust output regulation problem for nonlinear systems, IEEE Transactions on Automatic Control, vol. 46, no. 12, pp. 2028-2031, Dec. 2001.

[7] J. Huang, Nonlinear Output Regulation: Theory and Applications. Philadelphia, SIAM, 2004

[8] J. Huang and Z. Chen, "A general framework for tackling output regulation problem," IEEE Transactions on Automatic Control, vol. 49, no. 12, pp. 2203-2218, Dec. 2004.

[9] K. S. Narendra and A. M. Annaswamy, Stable Adaptive Systems, Englewood Cliffs, NJ: Printice-Hall, 1989.

[10] V. O. Nikiforov, "Adaptive non-linear tracking with complete compensation of unknown disturbances," European Journal of Control, vol. 4, pp.132-139, 1998.

[11] F. Delli Priscoli, L. Marconi, and A. Isidori, "A new approach to adaptive nonlinear regulation," SIAM J. Control Optim., vol. 45, no. 3, pp. 829-855, 2006.

[12] A. Serrani and A. Isidori, "Global robust output regulation for a class of nonlinear systems," Systems and Control Letters, vol. 39, pp. 133$139,2000$.

[13] A. Serrani, A. Isidori and L. Marconi, "Semiglobal nonlinear output regulation with adaptive internal model," IEEE Transactions on Automatic Control, vol. 46, no. 8, pp. 1178-1194, Aug. 2001.

[14] X. Ye and J. Huang, "Decentralized adaptive output regulation for a class of large-scale nonlinear systems," IEEE Transactions on Automatic Control, vol. 48, no. 2, pp. 276-281, Feb. 2003.

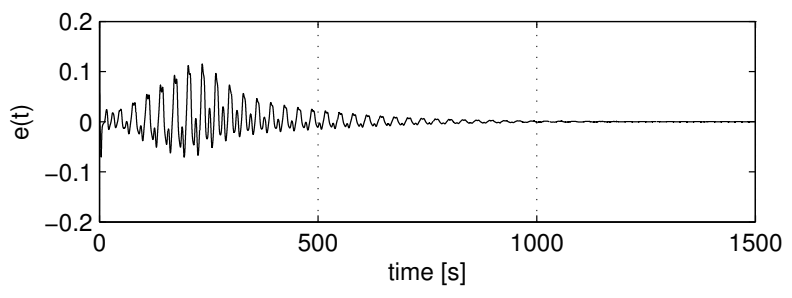

Fig. 1. Profile of the tracking error.

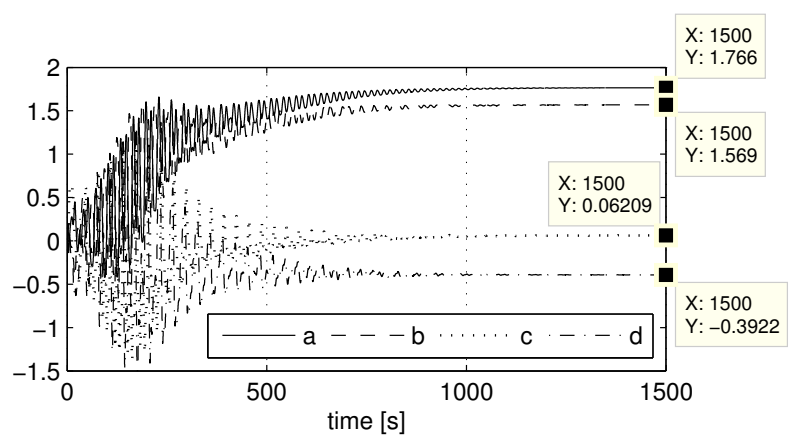

Fig. 2. Parameter convergence (a: $\hat{\Psi}_{1}$, b: $\hat{\Psi}_{2}$, c: $\hat{\Psi}_{3}$, d: $\hat{\Psi}_{4}$ ). 